\title{
How Can Health Care Professionals Communicate Effectively with Adolescent and Young Adults Who Have Completed Cancer Treatment? A Systematic Review
}

\author{
Luke A.M. Smith, BA (Hons), ${ }^{1}$ Deborah J. Critoph, MSc, ${ }^{2}$ and Helen M. Hatcher, $\mathrm{PhD}^{1,3,4}$
}

\begin{abstract}
Communication with teenage and young adult or adolescent and young adult (AYA) patients with cancer is critically important and not consistently taught in health care education. We have developed a local training for medical students to experience and join AYA cancer consultations to improve clinicians' abilities in the future. We have undertaken a systematic review of the literature, to provide a comprehensive overview of studies evaluating communication in this specific patient group, to guide clinical practice and future research. We searched MEDLINE, EMBASE, PsycINFO, Web of Science, and CINAHL databases for literature containing data relating to communication in AYAs previously treated for malignant disease. To ensure as comprehensive a review as possible, the researchers defined the AYA age range using the extremes used globally: 13-39 years. Two thousand eight hundred and thirty-seven articles were identified, which were reduced to 1517 after duplicates were removed. After handsearching references and citation tracking, 1556 abstracts were screened of which 40 full-text articles were assessed for eligibility. Only 12 met all inclusion criteria and were included in the final analysis. Young people want to be heard and have trust in their health care professionals (HCPs). They want to be involved in decisions about their care, and HCPs must establish who else the AYA wants to be involved in the decision-making process. AYAs want information to be given in an amount they can control and at a time preferred by them. Further education of communication skills, especially triadic communication, is essential for professionals involved in AYA care.
\end{abstract}

Keywords: communication skills, impact, triadic communication, long-term follow-up, survivorship

\section{Introduction}

$\mathbf{C}$ ANCER IS THE leading cause of nontraumatic death in adolescent and young adults (AYAs) in the United States and Europe. ${ }^{1-3}$ Each year, more than 11,000 AYA patients in the United States and over 2000 in the United Kingdom are diagnosed with cancer, and incidence rates are rising. ${ }^{2,4}$ It is increasingly recognized that such patients have experiences and communication needs that differ significantly from those of younger children and older adults. Besides having to negotiate the physical, cognitive, emotional, and behavioral changes that occur in adolescence, young people can suffer from a different cancer profile, longer periods of cancer treatment, a worse prognosis, and a particularly devastating sense of despair and isolation. ${ }^{5,6}$

There has been an international drive to develop ageappropriate specialist care provided by experienced health care professionals (HCPs) and to generate an evidence base addressing the specific needs of this patient group. If AYAs are not heard and understood they can be labelled as difficult or a challenge, which undoubtedly affects their future care and impedes shared decision making (SDM). We have been interested in investigating the role of age appropriate communication for these patients to aid them through their journey.

This review was designed to be broad to provide a comprehensive overview of studies investigating any aspect of AYA communication studies in survivors of cancer aged 13-39. The definition of AYA varies between countries and so, to try and capture the extent of research in this area, the researchers chose to encompass the lowest (13 years) and highest (39 years) ages when developing the research questions and search strategy. The research questions were:

\footnotetext{
${ }^{1}$ School of Clinical Medicine, University of Cambridge, Cambridge, United Kingdom.

${ }^{2}$ Clinical Communications Skills Department, School of Clinical Medicine, University of Cambridge, Cambridge, United Kingdom.

${ }^{3}$ Department of Oncology and ${ }^{4}$ TYA Cancer Service, Addenbrookes Hospital, Cambridge University Hospitals NHS Trust Foundation, Cambridge, United Kingdom.
} 


\begin{tabular}{|c|c|}
\hline $\begin{array}{l}\text { Strand } 1 \\
\text { AYA cancer }\end{array}$ & $\begin{array}{l}\text { TYA cancer or TYA oncology or } \\
\text { teenage and young adult adj5 cancer } \\
\text { or teenage and young adult adj5 } \\
\text { oncology or teenage* adj5 cancer or } \\
\text { teenage* adj5 oncology or } \\
\text { adolescen* adj } 5 \text { cancer or } \\
\text { adolescen* adj } 5 \text { oncology or young } \\
\text { people adj } 5 \text { cancer or young people } \\
\text { adj } 5 \text { oncology }\end{array}$ \\
\hline $\begin{array}{l}\text { Strand } 2 \\
\quad \text { Communication }\end{array}$ & $\begin{array}{l}\text { communicat* or discuss* or disclos* } \\
\text { or inform* }\end{array}$ \\
\hline $\begin{array}{l}\text { Strand } 3 \\
\quad \text { Survivorship }\end{array}$ & $\begin{array}{l}\text { suviv* or off treatment or post } \\
\text { treatment or long term follow up or } \\
\text { late effects }\end{array}$ \\
\hline Final search & 1 and 2 and 3 \\
\hline
\end{tabular}

adj5, adjacency function to search for words or terms in close proximity to each other in the text, in this case within five words (near function in some databases); AYA, adolescent and young adult; TYA, teenage and young adult.

(1) Is there anything written about HCP's communication with AYAs post-treatment and, if so, what are the communication skills required for a productive discussion with AYAs about their life after cancer?

(2) What is the impact of communication on AYA cancer patients in post-treatment follow-up and late effects clinics?

(3) What are the correlates of effective communication in this patient group?

(4) What cues do AYAs use to express their needs and concerns and are they different to other age groups?

(5) What is the impact of an AYA's supporters being present in discussions?

(6) How can HCPs give information effectively and promote SDM in this patient group?

\section{Methods}

\section{Literature search strategy}

A search of the literature was performed in March 2019 by L.A.M.S., the advice of a medical librarian was sought when developing the search strategy. Keywords were generated across three strands detailed in Table 1. To ensure a comprehensive search we preferentially used keywords over searching MeSH terms. Databases searched were Medline, Embase, PsycINFO, Web of Science, and CINAHL, supplemented by handsearching references and citing articles of relevant articles included in this review. Related systematic reviews were searched, including reviews evaluating the inclusion question criteria.

\section{Selection criteria}

The key inclusion criteria were that all study participants had previously had malignant disease and that either the majority was aged 13-39 years old at the time of the study or the results for this age group were presented separately. A third requirement was that the majority of participants in this age group were "off-treatment." Included studies could investigate any area of communication skills provided they focused on how to communicate effectively with AYAs.

Exclusion criteria included non-English language publications, systematic reviews, conference abstracts, or unpublished articles. Importantly, articles focusing on information needs rather than communication skills were excluded.

\section{Quality assessment and data analysis}

All articles, irrespective of relevance and quality, were included, if they met the search criteria.

The searches yielded 2837 titles. One thousand five hundred seventeen unique articles (after removing duplicates) were screened by one researcher (L.A.M.S.). Database results were supplemented by handsearching references and citing articles of articles included in the final review giving 1556 abstracts, which were reviewed by two researchers (L.A.M.S. and D.J.C.); 40 full articles were read by two researchers (L.A.M.S., D.J.C.), who excluded most of them on the basis of the participants' ages or insufficient focus on effective communication. All researchers read the remaining articles, with disagreements resolved by discussion. The final number of included articles was 12. A PRISMA diagram summarizing the search results is shown in Figure 1. The included articles are summarized in Table 2. A narrative synthesis was undertaken in relation to each of the research questions.

\section{Results}

Despite the attempt to incorporate research produced internationally by applying a broad age range, 9 of the 12 articles included exclusively contained participants aged 25 years or less when participating in the study. This represented four unique participant groups (due to multiple analyses). The remaining three articles included participants both under and over 25 years of age. Therefore, this skew toward the younger AYAs should be considered when interpreting the following results.

\section{Long-term follow-up}

Only one article, by Smits-Seemann et al., specifically addressed the barriers to AYAs accessing long-term followup (LTFU) care with some focus on the influence of communication. AYAs are unsure about the purpose of LTFU and how they should approach it. ${ }^{7}$ This is not helped by HCPs who are vague about recommendations long term for patients or even explicitly communicate to AYAs that follow-up care is not essential. ${ }^{7}$ This may further entrench AYAs' skepticism of follow-up care, especially if tests used did not aid in the initial diagnosis of the cancer. In these situations, AYAs cannot rationalize their use in the context of survivorship and LTFU. $^{7}$

Furthermore, AYAs may have to juggle follow-up care with other emerging responsibilities not expected of them during their treatment, such as a career or family, making traveling significant distances for follow-up not worthwhile for some. ${ }^{7}$ Finally, the fear of recurrence or receiving bad news may lead to AYAs to avoid places of care post-treatment. ${ }^{7}$

\section{Individuality}

At a time when they are developing an awareness of self, AYAs in the studies frequently discussed the need to be seen 


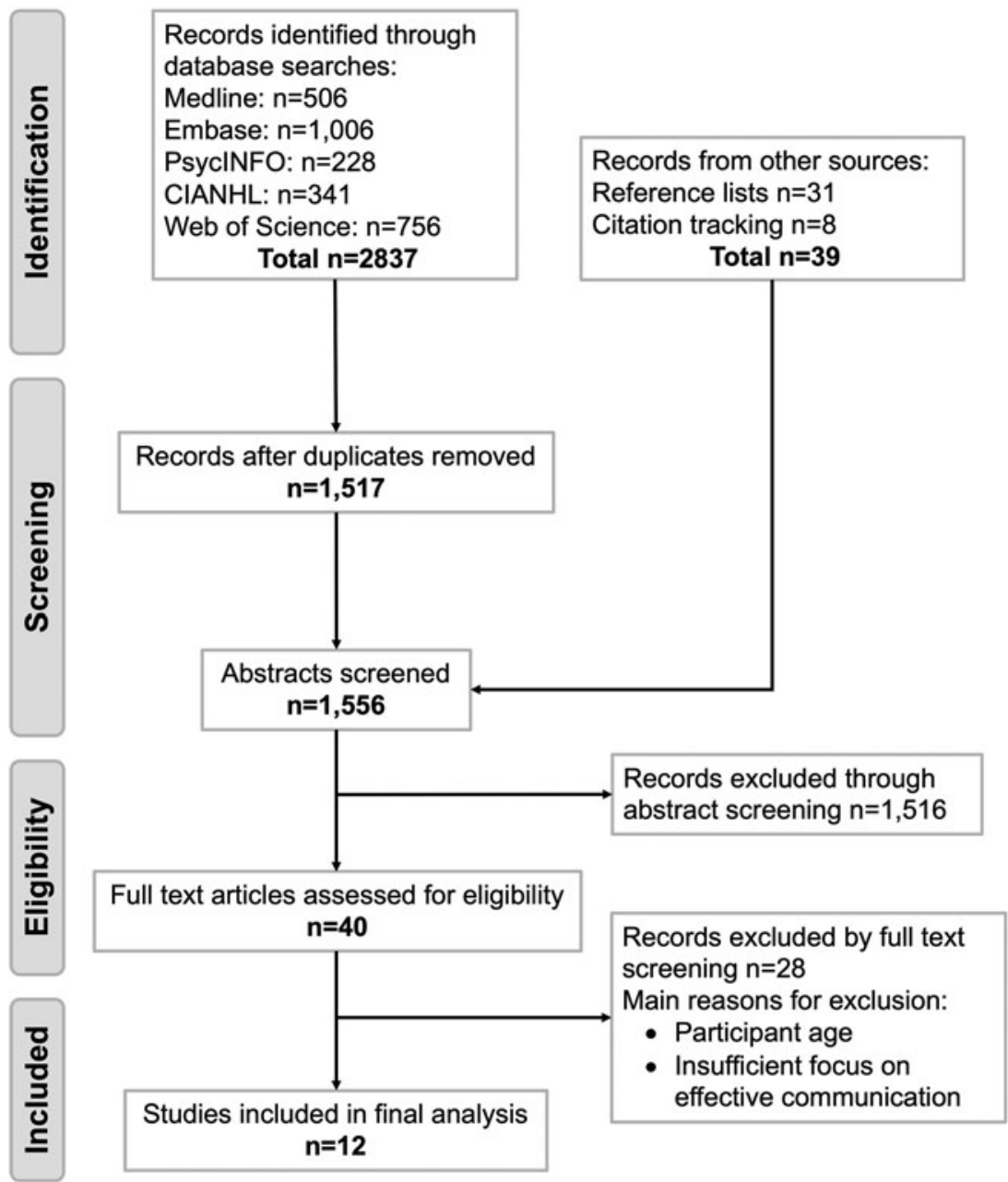

FIG. 1. PRISMA diagram of the search results.

as an individual, not just a "number," and be perceived as more than just their illness. ${ }^{8-11}$ Young people expressed a desire to stay connected with their "normal" life and want support from HCPs to do this and mitigate the impact of their cancer on their life. ${ }^{8,10,11}$ To that end, AYAs wanted HCPs to invest time in genuinely getting to know the AYA as a person. ${ }^{9}$ AYAs expressed the importance of their personal schedules and needs which, in their minds, take precedence. Thus, AYAs wanted their time to be well managed by HCPs. ${ }^{8}$ Belpame et al. explored the emergence of self-centeredness during cancer treatment, which is adopted by AYAs to help them endure the treatment. ${ }^{8}$

\section{Supporter(s)}

AYAs are often not alone, usually being accompanied by "supporter(s)", (parents, relatives, and/or significant others). ${ }^{8}$ This review only found examples examining the involvement of parents in conversations with no references to other types of supporter. In the Mellblom et al. series, parent(s) were present in $87 \%$ of consultations. ${ }^{12}$

The literature provides arguments both for the presence of parents and time alone for AYAs. AYAs themselves may have conflicting views regarding their parents: seeing parents being frequently present or immediately reachable as normal, while also feeling frustrated by their loss of independence and reliance on others. ${ }^{8}$ AYAs may believe themselves to be mature enough to make their own decisions, and the loyalties of HCPs can be tested when a younger AYA disagrees with their parent(s) who hold legal responsibility or when parents request for information to be withheld from AYAs. HCPs might find navigating these communication dynamics difficult. ${ }^{13,14}$

Conversely, rather than conflict, young people can display behaviors of deferral to their parents; at the extreme parents can "take over" the conversation, and some parents worried that their children deferred too much. ${ }^{14}$ However, parents can impact positively on communication with HCPs: for example, parents are often able to explain things in a way that makes sense to the AYA when HCPs use confusing language. ${ }^{14}$ Moreover, parents may be an important catalyst for discussion, initiating a similar proportion of conversations on late effects $(15.2 \%)$ compared with their child $(13.6 \%)^{15}$ and expressing around a quarter of cues/concerns in consultations. ${ }^{12}$

Equally, time alone appears beneficial for both AYAs and parents. Parents and AYAs may not fully disclose information when both parties are present: Hong et al. found that young people and their parents want to shield each other from information that might cause distress, ${ }^{14}$ and Mellblom et al. found that being alone was associated with greater expression 


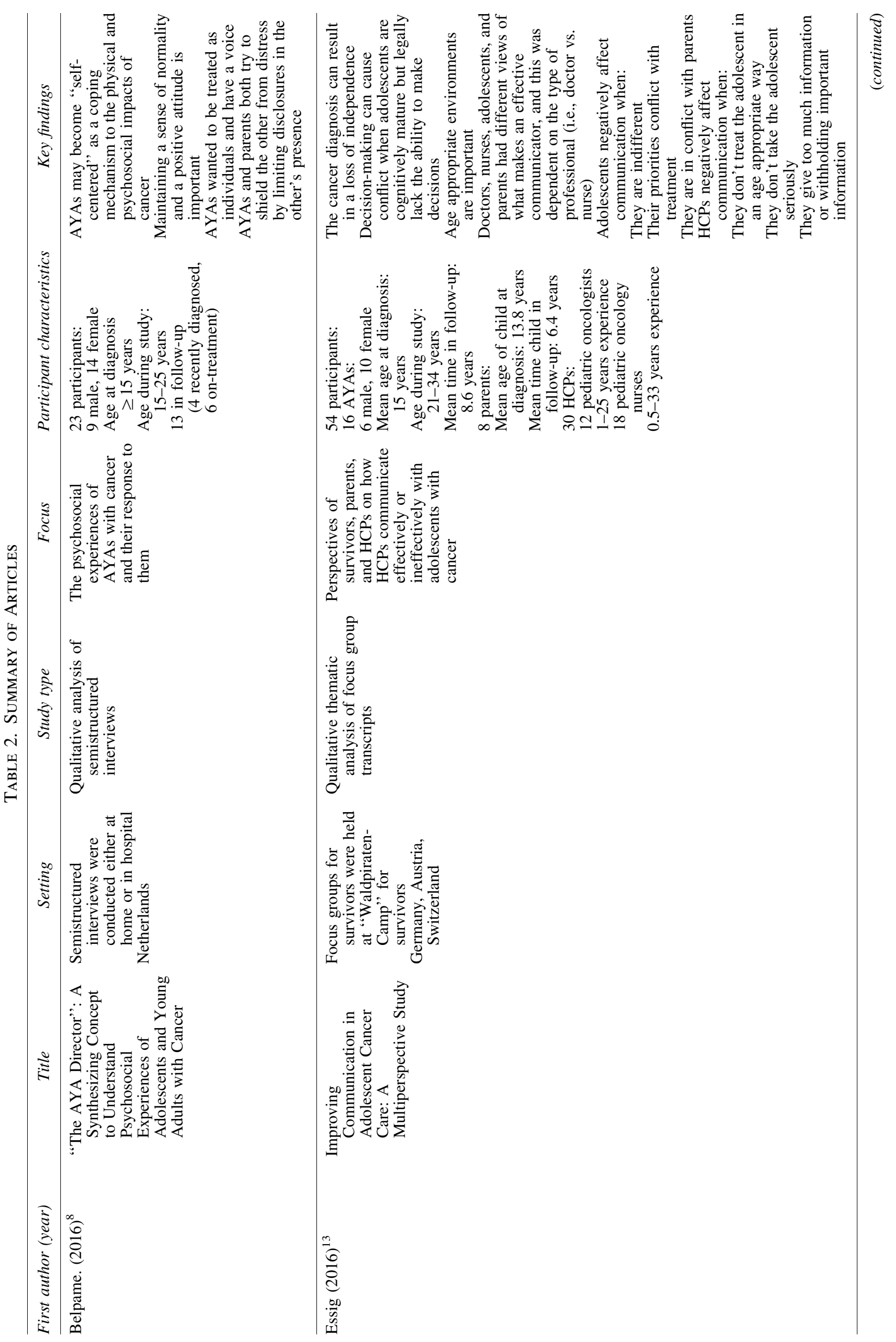




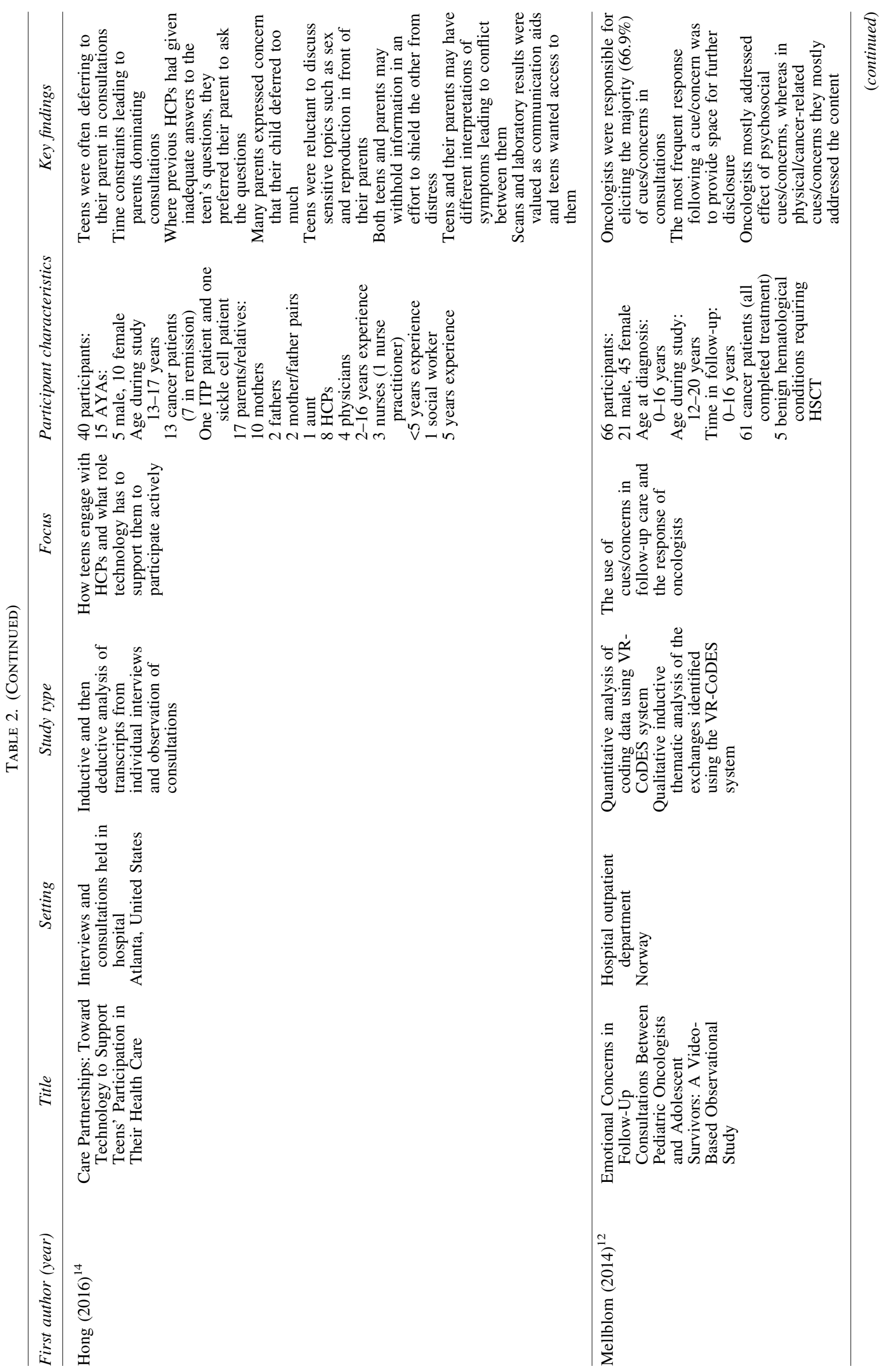




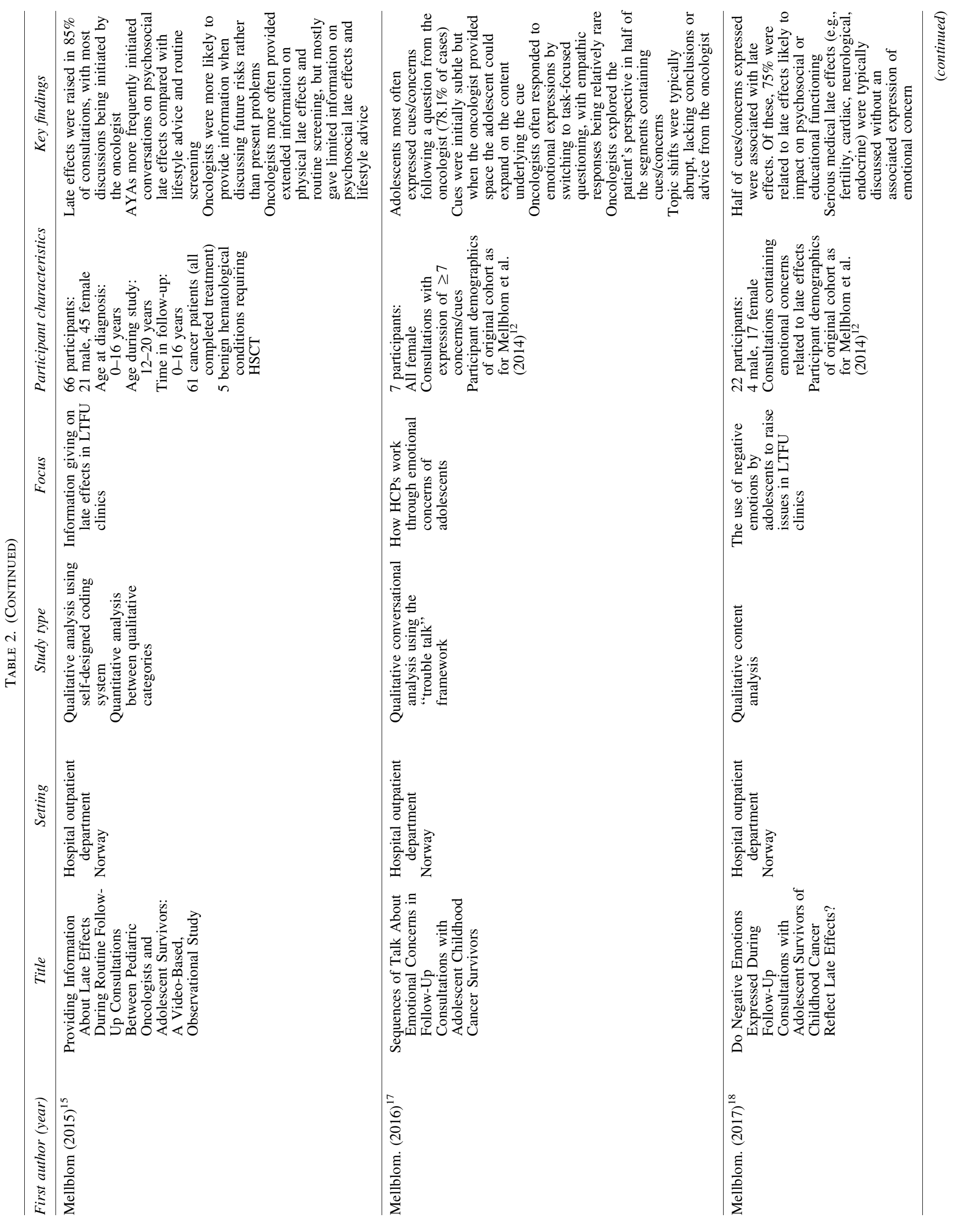




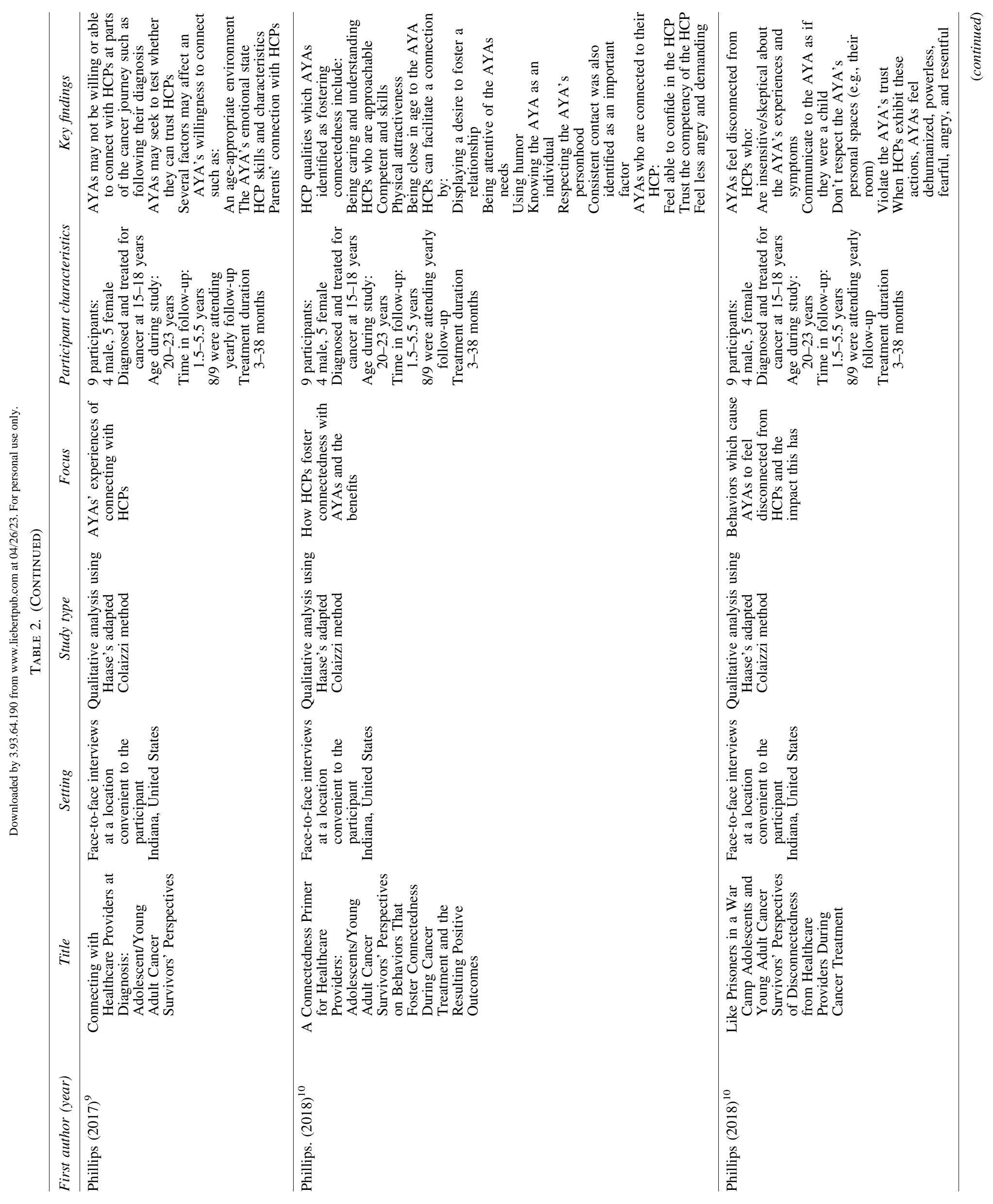




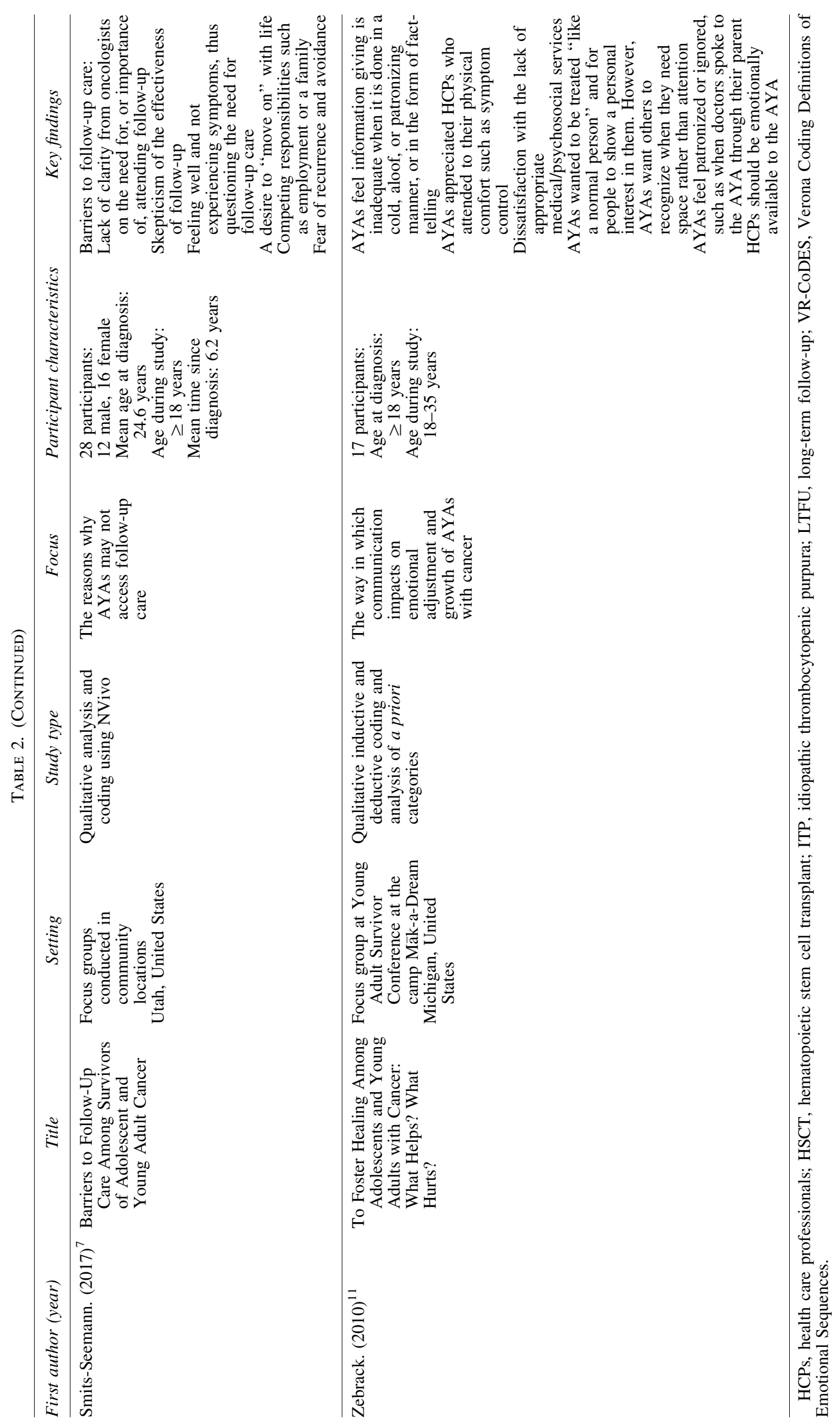


of cues/concerns. ${ }^{12}$ AYAs also recognized the need for private lines of communication ${ }^{14}$ and reflected that they confided in HCPs on matters they would not discuss with those close to them. ${ }^{8,10,14}$ Taken together, the evidence suggests that including parents in conversations is valuable, but that time alone provides AYAs necessary space for disclosure.

\section{Health care professionals}

The data give insight into qualities of HCPs which influence their interactions with AYAs and that AYAs recognize characteristics of an "ideal HCP." Moreover, AYAs may actively screen HCPs for the qualities of this ideal standard and even test the trust they place in HCPs. ${ }^{9}$ Throughout, there were recurring themes of a desire for HCPs who are empathic/thoughtful, acknowledging the gravity of the situation AYAs found them in and its impact on their life. $8,10,11,13$ Furthermore, HCPs who interact with AYAs should have professional competence specifically in AYA care, take AYAs seriously, and treat them like an adult. ${ }^{10,13}$ The desire for professional competence was balanced by the desire to have HCPs who adopt "a human approach"' showing genuine interest in the AYA. ${ }^{8-11,13}$

Humor was raised as a communication tool to help foster the relationship between AYAs and HCPs. ${ }^{9,10}$ AYAs were also forthcoming about the qualities of HCPs, which negatively affected the relationship. HCPs who seemed not to care about the AYA's situation or were awkwardly intrusive and pushy were viewed negatively. ${ }^{8,9,16}$ HCPs who treated AYAs like children or didn't take them seriously (e.g., skepticism of symptoms) lost the AYA's trust. ${ }^{9,13,16}$ HCPs speaking directly to/through parents leave AYAs feeling left out of their health care conversations. ${ }^{9,11}$ Moreover, a parent's own need to connect with HCPs may impede AYAs from forming a connection. ${ }^{9}$

\section{Cues}

A body of work by Mellblom et al. explored communication of emotional concerns and late effects in follow-up consultations of adolescent cancer survivors, by performing several analyses of recordings from an outpatient oncology follow-up clinic. ${ }^{12,15,17,18}$ In half of consultations, no cues/concerns were expressed and in consultations where cues/concerns were expressed, most were initiated by oncologists $(66.9 \%) .{ }^{12}$ Participant characteristics did not significantly differ between the consultations with and those without cues/ concerns. However, if cues/concerns were expressed, being alone was associated with greater expression levels. ${ }^{12}$

About half of emotional concerns were related to potential late effects, but the majority (75\%) reflected psychosocial issues rather than those related to serious medical late effects. While the latter were discussed in consultations, they were rarely in the context of emotional concern. ${ }^{18}$ Furthermore, investigation of conversations on late effects showed that $>70 \%$ were initiated by the oncologist and conversations related to lifestyle advice/routine screening almost exclusively initiated by oncologists. Conversely, psychosocial late effects were initiated least often by the oncologist $(52 \%)$, being initiated by patients and parents $28 \%$ and $20 \%$ of the time, respectively, the highest for any group of cues/concerns. ${ }^{15}$

Following a cue or concern, the predominant response from HCPs was to provide space for further disclosure of the cue/concern (76.5\%). ${ }^{12}$ Notably, HCPs were more likely to address the emotion of a psychosocial cue/concern compared with addressing the content of physical and cancer-related cues/concerns. ${ }^{12}$ In a small subsample of patients who expressed high numbers of cues/concerns, Mellblom et al. undertook conversational analysis using Jefferson's 'trouble talk" framework. ${ }^{17}$ They found that oncologists often respond to emotional expression with task-focused follow-up questioning without explicit reference to the emotion, and empathic responses were relatively rare. Even when emotional concerns were elaborated upon, abrupt topic shifts usually occurred without reaching a conclusion, usually after a significant pause. ${ }^{17}$

\section{Information giving}

Information giving by HCPs may be inadequate for several reasons. First, AYAs find it difficult navigating the complex milieu of medical jargon used by HCPs or are overwhelmed by the level of detail provided. ${ }^{8,13,14}$ Conversely, AYAs are dissatisfied when HCPs are vague when answering their questions. ${ }^{7}$ AYAs found information presented in an abrupt or matter-of-fact manner difficult to handle. ${ }^{8,11}$ AYAs wanted information to be made meaningful by contextualizing it to their way of life and information to focus on the present rather than future consequences. ${ }^{8}$ Despite this, HCPs often provided extended information on potential physical late effects and routine screening but gave little/no information on current problems, lifestyle advice, and potential psychosocial late effects. ${ }^{15}$

Helpful techniques HCPs used included using visual aids (imaging) and trend data (serial laboratory results), which AYAs found easier to correlate with their symptoms and personal experiences, ${ }^{14}$ and having control in advance of how much information they received. ${ }^{8}$ AYAs don't like to be spoken to through parents ${ }^{11}$ but may defer to parents when their previous experiences show that their parents receive more comprehensive answers to questions. ${ }^{14}$ Parents can explain answers using language the AYA can understand. ${ }^{14}$

\section{Shared decision making}

The studies suggest that AYA preferences for the level of involvement in SDM vary between individual AYAs, but AYAs want to have their voice heard and taken seriously and have their preferences respected. ${ }^{8,10,13}$ AYAs' priorities may conflict with treatment, which may precipitate communication difficulties with HCPs. ${ }^{13}$ However, AYAs may not have the skills to advocate for themselves or feel they don't have authority to overrule HCPs' views. ${ }^{16}$ HCP awareness here is paramount because AYAs want support from HCPs in maintaining their autonomy and independence. ${ }^{10}$ Studies highlighted the difficulty AYAs faced being spoken to like a child or in a patronizing manner ${ }^{8,11,13,16}$ and HCPs using language which was confusing or overwhelming, ${ }^{8,13,14}$ impacting on the AYA's participation. ${ }^{14}$

Moreover, in discussions, AYAs wanted HCPs to be honest and did not appreciate them withholding information. ${ }^{10,13,14}$ AYAs need the rationale of an examination, test, or procedure to be communicated as they felt things were being done to them needlessly. ${ }^{7,16}$ When their autonomy isn't respected, AYAs can be left feeling violated, objectified, and dehumanized. ${ }^{16}$ In response to difficult situations, AYAs may become withdrawn, apathetic, or passive aggressive in an attempt to dissociate $^{13,16}$ and AYAs expressed that their own emotional 
states (such as grumpiness or irritability) could make HCPs less willing to engage with them, ${ }^{9}$ which may impact on their ability to participate in health care discussions.

\section{Discussion}

Cancer has a profound impact on an AYA's life. They lose crucial formative years to multimodal treatment, numerous appointments, repeated admissions, and an uncertain future.

While the treatment phase can be highly demanding physically, socially, and emotionally, it is stereotypically very structured and AYAs have access to HCPs with specialist skills in AYA cancer care. It is therefore not surprising that AYAs may struggle at the end of treatment, where there can be a relatively abrupt entry into the vastness of survivorship and repatriation to community and primary care services. ${ }^{19,20}$ This may be exacerbated by patients being told that they can return to their "normal lives" 21 when many may find it difficult to reach common milestones of young people. ${ }^{22}$ As one patient reflected "Cancer has been my job... I' $m$ not sure how I'll be trying to get back into normal life again., ${ }^{23}$ HCPs must navigate this complex time and somehow support AYAs to transition to independence-an outcome of follow-up care. ${ }^{5,20}$

HCPs need to be open with AYAs about the difficulties of the post-treatment period and provide ongoing practical support. ${ }^{21}$ A key component of survivorship is recognizing the journey that patients have been on, not solely focusing on where they are going. This is consistent with our findings of repeated references in these studies to being treated as an individual, being cared for, and being seen as more than just a number or illness.

Two subgroups of patients exist within survivorshipthose diagnosed and treated for pediatric cancers who enter AYA services as survivors and those who were diagnosed and treated as AYAs. This may impact on their knowledge and reflections of their cancer journey, physical impacts of treatment, and psychosocial sequelae. Indeed, Kazak et al found that survivors diagnosed during adolescence had significantly more post-traumatic stress disorder symptoms, lower psychosocial health-related quality of life, and lower health perceptions and cognitive competence compared to survivors diagnosed at younger ages. ${ }^{24}$ Moreover, in younger cohorts, those around the patient may shoulder much of the psychological burden which could explain why family members, rather than survivors, are more dissatisfied with the information received post-treatment. ${ }^{21}$

Importantly, young people are often unsure who to approach about ongoing health problems, feeling that their concerns are too trivial to take to their oncologist over the course of treatment. ${ }^{23}$ AYAs may desire the support/input of their oncologist, a specialist who they have developed a relationship with over the course of their treatment. Furthermore, they may lack confidence in the abilities of their primary care practitioner to meet their complex needs, especially if there was delayed diagnosis of their cancer. HCPs should be mindful that AYA patients may not express cues and concerns as frequently or in the same way as older patient groups, ${ }^{12}$ and HCPs may need to be proactive at screening for issues in follow-up consultations. Moreover, HCPs should consider offering services AYAs rebuffed during treatment, such as counseling. AYAs may be more receptive to these services driven by a desire for assistance in dealing with what they have gone through and getting on with their lives. ${ }^{25}$

The relationship between the patient and treating clinician is clearly important. In the literature this is expressed in different ways, including "connectedness" and "therapeutic relationship." ${ }^{, 5,9,10,26}$ AYAs want to develop a personal relationship with their HCP: to feel known and cared for by someone they trust. The impact of this was highlighted by Phillips and Haase: AYAs felt this relationship helped to make them more tolerant of HCPs, the health care system, and its misgivings. This was not just confined to the HCP in question but seemed to have wider effects. ${ }^{10}$ Other studies reported that the relationship with their HCP allowed them to feel comfortable disclosing sensitive information or discussing difficult topics. ${ }^{8,10}$ It would also allow HCPs to understand the decisions that AYAs make. Previous work has explored why adolescents may undertake behaviors that adults may deem risky despite perceiving risk in the same way as adults. ${ }^{27}$ Indeed, AYAs may incorporate a variety of social and emotional influences into their decision-making process rather than an "objective" evaluation of risk and consequence. HCPs must be mindful not to conflate this with a lack of understanding, and AYAs may feel their decisions are entirely justified and reasonable. ${ }^{27}$ However, despite the perceived benefits of developing a relationship from the perspective of the AYA, there may be some hesitancy from HCPs about maintaining a professional boundary and not being "friends" with patients. Therefore, more work should be done to develop ways in fostering a positive relationship while keeping both parties safe. Simple steps such as conveying empathy, care, and understanding may go a long way to improving AYAs' experiences without crossing any professional boundaries. It is also important to recognize the actions of HCPs that create rifts between HCPs and AYAs. ${ }^{16}$ The type of relationship AYAs seek may differ from AYA to AYA but also between professionals (e.g., doctors versus nurses) ${ }^{13}$ It is paramount that all HCPs working with AYAs develop the relevant skills.

Supporters accompanying AYAs undoubtedly influence communication dynamics in AYA cancer care. The wider literature describes the presence of a third party in medical consultations as the triadic consultation, with research dedicated to communication in this setting. Consultations with three (or more) participants are more complex and challenging for HCPs to navigate, and thus, specific training is warranted. In the context of illness, young adults are often pulled back into the safety of the parental nest and their physical limitations may mandate the family to revert to an earlier dynamic, with parents assuming an overprotective role. ${ }^{28,29}$ However, there is insufficient research on the interactional dynamics in triadic consultations and the skills required for a successful and effective encounter. ${ }^{30}$ LaidsaarPowell et al. conducted a systematic review of triadic medical consultations, which indicated that companions regularly attended consultations and patients with higher needs were more often accompanied. Supporters assumed a variety of roles and adopted behaviors, which could be perceived as both helpful (e.g., informational support) and unhelpful (e.g., dominating/demanding behaviors). ${ }^{31}$ Their involvement often raised challenges, and the supporters' preferences for involvement varied widely. AYAs desire time alone with HCPs, for example, to discuss sexual and reproductive health. ${ }^{32}$ 
Despite this, HCPs do not routinely ask parents to leave the room for such discussions ${ }^{33}$ and, thus, do not have time alone with AYA patients. HCPs stated that they find asking parents to step out difficult, and some reported that parents were distressed about the potential conversation content in their absence. ${ }^{33}$ In a study conducted by Olsson et al., participants expressed the need to talk with the physician with and without relatives, wanting HCPs to make time alone a routine practice. ${ }^{25}$

Gibson et al. propose that an AYA shifts positions with their parent(s) and only adopts the role of primary communicator in consultations once autonomy has been gained (or regained). ${ }^{34}$ It can be argued that this is a key goal in AYA cancer care, particularly transitioning to self-management in survivorship. Notably, the more involved parents are in care, the less control and interest adolescents have in their own disease management. ${ }^{35}$ For young adult cancer survivors, a history of familial decision-making during cancer treatment may complicate the transition of roles played by the AYA, the family, and the doctor in adulthood. ${ }^{36}$ The studies in this review suggest that AYAs' preferences on the degree of pa- rental involvement are dependent on the individual and may change throughout the cancer trajectory. The impact of cancer symptoms, psychological or emotional stress, lack of rapport, or inadequate HCP communication may interfere with AYAs' desire to communicate ${ }^{7-11,13,14,16}$ and may ultimately result in deferral to parents. ${ }^{14}$ AYA specialists must be cognizant of these complex challenges within triadic communication to promote active participation by AYAs in SDM. Determining when and how to involve young people is complex; it requires navigation of the triad factoring the AYA's desire to have discussions both with and without the presence of their supporters. ${ }^{37}$ Each AYA's cancer story is unique and exploring how they experienced cancer, its treatments, and what their cancer trajectory means to them is more likely to enable individualized care $^{38}$ and give AYAs a sense of agency and increase concordance. ${ }^{39}$ Triadic communication is a key feature of AYA cancer care. Third parties can be a help and a hindrance, so it is essential that HCPs develop strategies to successfully navigate triadic interviews, enabling and supporting the AYAs' participation.

Table 3. Recommendations for Clinical Practice

Core communication skills

AYA specific communication skills

\section{Establishing rapport}

Speak directly to the patient, acknowledge their supporters but keep the patient at the center of care
Take an active interest in the AYA's life beyond their cancer experience. It may vary for different ages: in younger AYAs this could be school/college/hobbies/interests and in older AYAs, career/family

Keep trying to engage with AYAs where disengagement or passive aggression is a barrier to effective communication

For younger AYAs be mindful to not talk to them as if they are a child and to not patronize them

Navigating the conversation

Be vigilant for verbal and nonverbal cues

Acknowledge and explore patient concerns

AYAs may not utilize cues in the same way as other age groups. Nonverbal cues are more common than verbal-be alert for them

Spend time alone with the AYA to discuss sensitive topics

Once rapport is established, humor may be a useful tool to build the professional relationship
Information giving and shared decision-making

Use language that the patient can understand

Explore the patient's agenda and priorities

Assess the patient's wishes for the amount of information they want to receive, and their level of involvement in decisionmaking

Be honest, do not withhold information
AYAs, depending on their age, may bring different types of supporter (e.g., parent vs. partner) who may have different agendas/needs

Use scans, laboratory results, and diagrams, where available, as communication aids

Contextualize information to the AYA's life

Check the AYA's understanding; for younger AYAs, parents may be able to frame information in a way which is easier for the AYA to process

Revisit an AYA's preferences for information giving and decision-making periodically

Facilitating engagement in LTFU

Address barriers to attendance

Encourage self-management, be opportunistic with health promotion education
For AYAs treated in pediatrics where information may have been directed toward parents, it is important to establish the AYA's "starting point": Understanding of diagnosis

Treatment received, and

Potential late effects

Assess the AYA's developmental, emotional, and mental health. This may be facilitated by the use of a psychosocial assessment tool

Bridge information gaps based on the AYA's starting point. Use this opportunity to emphasize the importance/need for LTFU

Explain the rationale behind tests in LTFU

Written treatment summaries are useful to support verbal communication 


\section{Strengths and limitations}

Our systematic review, which included a systematic and broad search strategy with electronic and manual searching of the literature, has strengths and limitations. It provides a unique perspective on communication in AYA care with the inclusion criteria permitting the extensive variety of professionals characteristic of this specialty. It is important to note that the studies included were small samples and all qualitative in nature, although this is to be expected in such a unique treatment population. There were multiple analyses of some data sets meaning that for the 12 articles included, there were 7 unique participant groups. Moreover, some aspects (namely barriers to LTFU and cues/concerns) were studied in a single participant group; this strengthens the case for further research, but means that the results may not be more widely applicable. Moreover, despite the objective of this review to encompass all definitions of the AYA range globally (i.e., 13-39), due to the final studies included, younger AYAs $(<25$ years) may be overrepresented in the findings of this review. While we feel the recommendations for practice are applicable to all HCPs, the data on which they are based are confined to doctors and nurses.

\section{Implications for clinical practice}

Recommendations for practice. Communication with AYAs can be nuanced and challenging, even for experienced communicators. What is clear is that building rapport with AYAs may be hard won but the payoff is great, and HCPs should not take an AYA's silence as satisfaction. These recommendations for practice represent a synthesis of the findings from the literature as outlined in Table 3. Some of the elements drawn from the literature are applicable to all patients, not just AYAs, and some may seem as "core skills." However, it is important to note that Table 3 represents elements of communication felt to be lacking by HCPs, as qualified by the AYA participants in the studies of this review. Table 3 delineates between core communication skills and AYA specific strategies to aid HCPs further. Therefore, Table 3 is relevant to various HCPs and can be easily implemented into HCPs daily practice, including for information giving, decision-making, and LTFU care more widely. Importantly, relationship building is a longitudinal process and not all these recommendations will be relevant in every interaction. Parallel to this, AYAs themselves will be transitioning through milestones in multiple domains (socially, financially, developmentally, and psychologically). HCPs should be cognizant of the need to reassess the preferences of AYAs periodically.

Due to the age and global distribution of participants, we feel they should provide a useful checklist; however, as previously discussed, there may be more relevant for younger AYAs. Where appropriate, references have been made in Table 3 to potential areas of difference between younger and older AYAs. In addition, it should be noted that there is medicolegal variation across the countries where these studies were conducted. It is outside of the scope of this review to explore this topic extensively, but these recommendations should be incorporated in accordance with local legal guidance.

\section{Implications for future research}

The small number of studies in this review highlights the need for further research into communication skills to inform the practices of professionals working with AYA cancer survivors. While some areas of communication skills may be generalizable to all of AYA cancer care, other additional skills may be required for different phases of the cancer trajectory, such as in survivorship, and this may be specific to a profession. Moreover, geographical and cultural differences may influence the skills required, which need to be explored further. More work needs to be done on what communications skills development professionals who work with AYA patient groups require. This needs to be supported by evidence-based training programs to facilitate professional development.

\section{Conclusions}

AYAs are a unique group of patients transitioning to independence. They need their HCPs to listen to them and those working with AYAs affected by cancer must understand the importance of appreciating an AYA's individual perspective to be able to fully engage the AYA in their health care. In doing so HCPs can ascertain the AYAs' preferences for information giving and decision-making. Triadic communication, as highlighted, is a key feature in AYA care, and professionals need specific training in the skills required to navigate the unique challenges it raises. HCPs can have a profound impact on an AYA's view of, and engagement with, health care. It is the responsibility of those working with AYAs, regardless of the setting, to ensure that they have the appropriate skill set.

\section{Author Disclosure Statement}

No competing financial interests exist.

\section{Funding Information}

No funding was received for this article.

\section{References}

1. O'Hara C, Moran A; TYA National Cancer Intelligence Advisory Group. Survival in teenagers and young adults with cancer in the UK. London: National Cancer Intelligence Network; 2012.

2. Bleyer A, O'Leary M, Barr R, Ries LAG (Eds). Cancer epidemiology in older adolescents and young adults 15 to 29 years of age, including SEER incidence and survival: 1975-2000. Bethesda, MD: National Cancer Institute; 2006.

3. Office for National Statistics. Mortality statistics: deaths registered in England and Wales, DR series. London, UK: Office for National Statistics; 2012.

4. Cancer Research UK. Young peoples' cancers incidence statistics [Internet]. Accessed July 10, 2019 from: https://www .cancerresearchuk.org/health-professional/cancer-statistics/ young-people-cancers/incidence

5. Smith S, Case L, Waterhouse K, et al. A blueprint of care for teenagers and young adults with cancer. London: Teenage Cancer Trust; 2012.

6. National Institute for Health and Clinical Excellence. Guidance on improving outcomes in children and young people with cancer. London: NICE; 2005.

7. Smits-Seemann RR, Kaul S, Zamora ER, et al. Barriers to follow-up care among survivors of adolescent and young adult cancer. J Cancer Surviv. 2017;11(1):126-32. 
8. Belpame N, Kars MC, Beeckman D, et al. "The AYA Director": a synthesizing concept to understand psychosocial experiences of adolescents and young adults with cancer. Cancer Nurs. 2016;39(4):292-302.

9. Phillips CR, Haase JE, Broome ME, et al. Connecting with healthcare providers at diagnosis: adolescent/young adult cancer survivors' perspectives. Int J Qual Stud Health Well-being. 2017;12(1):1325699.

10. Phillips CR, Haase JE. A connectedness primer for healthcare providers: adolescents/young adult cancer survivors' perspectives on behaviors that foster connectedness during cancer treatment and the resulting positive outcomes. J Adolesc Young Adult Oncol. 2018;7(2): 174-80.

11. Zebrack B, Chesler MA, Kaplan S. To foster healing among adolescents and young adults with cancer: what helps? What hurts? Support Care Cancer. 2010;18(1):131-5.

12. Mellblom AV, Finset A, Korsvold L, et al. Emotional concerns in follow-up consultations between paediatric oncologists and adolescent survivors: a video-based observational study. Psychooncology. 2014;23(12):1365-72.

13. Essig S, Steiner C, Kuehni CE, et al. Improving communication in adolescent cancer care: a multiperspective study. Pediatr Blood Cancer. 2016;63(8):1423-30.

14. Hong MK, Wilcox L, Machado D, et al. Care partnerships: toward technology to support teens' participation in their health care. Proc SIGCHI Conf Hum Factors Comput Syst CHI Conf. 2016;2016:5337-49.

15. Mellblom AV, Korsvold L, Finset A, et al. Providing information about late effects during routine follow-up consultations between pediatric oncologists and adolescent survivors: a video-based, observational study. J Adolesc Young Adult Oncol. 2015;4(4):200-8.

16. Phillips CR, Haase JE. Like prisoners in a war camp adolescents and young adult cancer survivors' perspectives of disconnectedness from healthcare providers during cancer treatment. Cancer Nurs. 2020;43(1):69-77.

17. Mellblom AV, Korsvold L, Ruud E, et al. Sequences of talk about emotional concerns in follow-up consultations with adolescent childhood cancer survivors. Patient Educ Couns. 2016;99(1):77-84.

18. Mellblom AV, Ruud E, Loge JH, Lie HC. Do negative emotions expressed during follow-up consultations with adolescent survivors of childhood cancer reflect late effects? Patient Educ Couns. 2017;100(11):2098101.

19. Patterson P, McDonald FEJ, Zebrack B, Medlow S. Emerging issues among adolescent and young adult cancer survivors. Semin Oncol Nurs. 2015;31(1):53-9.

20. CanTeen. Exploring survivorship care for adolescent and young adult cancer survivors in Australia. Australia: CanTeen; 2015.

21. Wakefield CE, Butow P, Fleming CAK, et al. Family information needs at childhood cancer treatment completion. Pediatr Blood Cancer. 2012;58(4):621-6.

22. Wong AWK, Chang TT, Christopher K, et al. Patterns of unmet needs in adolescent and young adult (AYA) cancer survivors: in their own words. J Cancer Surviv. 2017;11(6): 751-64.

23. Thompson K, Palmer S, Dyson G. Adolescents \& young adults: issues in transition from active therapy into followup care. Eur J Oncol Nurs. 2009;13(3):207-12.

24. Kazak AE, DeRosa BW, Schwartz LA, et al. Psychological outcomes and health beliefs in adolescent and young adult survivors of childhood cancer and controls. J Clin Oncol. 2010;28(12):2002-7.

25. Olsson M, Jarfelt M, Pergert P, Enskär K. Experiences of teenagers and young adults treated for cancer in Sweden. Eur J Oncol Nurs. 2015;19(5):575-81.

26. Ritchie MA. Psychosocial nursing care for adolescents with cancer. Issues Compr Pediatr Nurs. 2001;24(January):165-75.

27. Steinberg L. Risk taking in adolescence: what changes, and why? Ann N Y Acad Sci. 2004;1021(1):51-8.

28. Hinds PS, Quargnenti AG, Wentz T. Measuring symptom distress in adolescents with cancer. J Paediatr Oncol Nurs. 1992;9(2):84-6.

29. Grinyer A. Young people living with cancer: implications for policy and practice. Open University Press: Maidenhead, England; 2007; 200 p.

30. Tates K, Meeuwesen L. Doctor-parent-child communication. A (re)view of the literature. Soc Sci Med. 2001;52:839-51.

31. Laidsaar-Powell RC, Butow PN, Bu S, et al. Physicianpatient-companion communication and decision-making: a systematic review of triadic medical consultations. Patient Educ Couns. 2013;91:3-13.

32. Frederick NN, Revette A, Michaud A, Bober SL. A qualitative study of sexual and reproductive health communication with adolescent and young adult oncology patients. Pediatr Blood Cancer. 2019;66:e27673.

33. Frederick NN, Campbell K, Kenney LB, et al. Barriers and facilitators to sexual and reproductive health communication between pediatric oncology clinicians and adolescent and young adult patients: the clinician perspective. Pediatr Blood Cancer. 2018;65(8):9.

34. Gibson F, Aldiss S, Horstman M, et al. Children and young people's experiences of cancer care: a qualitative research study using participatory methods. Int J Nurs Stud. 2010; 47(11):1397-407.

35. Huang JS, Gottschalk M, Pian M, et al. Transition to adult care: systematic assessment of adolescents with chronic illnesses and their medical teams. J Pediatr. 2011;159(6): 994.e2-8.e2.

36. Shay LA, Schmidt S, Cornell SD, Parsons HM. "Making my own decisions sometimes": a pilot study of young adult cancer survivors' perspectives on medical decision-making. J Cancer Educ. 2018;33(6):1341-6.

37. Kent EE, Parry C, Montoya MJ, et al. "You're too young for this": adolescent and young adults' perspectives on cancer survivorship. J Psychosoc Oncol. 2012;30(2):260-79.

38. Kelly D, Gibson F (Eds). Developing an integrated approach to the care of adolescents and young adults with cancer. In: Cancer care for adolescents and young adults. Wiley Online Books; 2008; pp. 229-247.

39. Robertson E, Wakefield C, Marshall K, Sansom-Daly U. Strategies to improve adherence to treatment in adolescents and young adults with cancer: a systematic review. Clin Oncol Adolesc Young Adults. 2015;5:35.

Address correspondence to: Deborah J. Critoph, $M S c$ Clinical Communications Skills Department School of Clinical Medicine University of Cambridge Box 111

Cambridge CB2 OSP United Kingdom

Email: dc625@medschl.cam.ac.uk 